\title{
Linear-polarization dependence of microwave-induced magnetoresistance oscillations in high-mobility two-dimensional systems
}

\author{
X. L. Lei and S. Y. Liu \\ Key Laboratory of Artificial Structures and Quantum Control, Department of Physics, \\ Shanghai Jiao Tong University, 800 Dongchuan Road, Shanghai 200240, China
}

\begin{abstract}
We examine the effect of changing the linear polarization angle $\theta$ of incident microwaves with respect to the dc current on radiation-induced magnetoresistance oscillations in a two-dimensional (2D) system within the balance-equation formulation of the photon-assisted magnetotransport model, considering the radiative decay as the sole damping mechanism. At an extremum the amplitude of oscillatory magnetoresistance $R_{x x}$ exhibits a sinusoidal, up to a factor of 5 , magnitude variation with rotating the polarization angle $\theta$. The maximal amplitude shows up generally at a nonzero $\theta$, which is dependent upon the extremum in question, the $2 \mathrm{D}$ electron setup, the radiation frequency and the magnetic field orientation. These results provide a natural explanation for the experimental observations by Mani et al. [Phys. Rev. B 84, 085308 (2011)], and Ramanayaka et al. [Phys. Rev. B 85, 205315 (2012)].
\end{abstract}

PACS numbers: 73.50.Jt, 73.40.-c, 73.43.Qt, 71.70.Di

\section{INTRODUCTION}

Microwave induced magnetoresistance oscillation in high-mobility two-dimensional (2D) electron systems has been a subject of intensive experimental $1-18$ and theoretical ${ }^{19}-35$ studies over the past decades. Though a consensus on the period and phase of the oscillation has long been reached, the effect of the microwave polarization on the amplitude of the oscillation remains one of the most challenging and unsolved issues since the discovery of microwave-induced magnetoresistance oscillations.

Early measurement on L-shaped Hall bars indicated that the period and phase of radiation-induced magnetoresistance oscillations are insensitive to the relative orientation between the microwave polarization and the current. $\underline{9}$ A later experiment $\frac{13}{}$ carried out on specimens with a square geometry in a quasioptical setup reported a striking result that not only the frequency and phase but also the amplitude of radiation-induced resistance oscillations and the zero resistance regions are notably immune to the sense of circular and linear polarizations of microwaves. This influential result raised a big challenge to the existing theoretical models, in which, though no detailed investigation was reported, some kind of polarization dependence was believed to exist,$\frac{19,22,23}{2}$ and thus expedited the emergence of different scenarios capable of showing polarization immunity of microwave magnetoresistance response ${ }^{33.34}$ In a recent study, Mani et al ${ }^{17}$ found a strong sensitivity in the amplitude of radiationinduced magnetoresistance oscillations to the relative orientation of the linear polarization with respect to the Hall bar axis. Particularly, more detailed measurement $\frac{18}{\underline{6}}$ by rotating, by an angle $\theta$, the polarization direction of linearly polarized microwaves with respect to the long axis of the Hall bar electron devices, showed, at relatively low microwave power, a strong sinusoidal variation in the diagonal resistance $R_{x x}$ vs $\theta$ at the oscillatory extrema. And, unexpectedly, the angle for the maximal oscillatory
$R_{x x}$ response under a given-power linear-polarized microwave, which is not at $0^{\circ}$ or $90^{\circ}$, appears to depend upon the radiation frequency, the extremum in question, and the magnetic field orientation. So far, there has been no theoretical explanation for these interesting observations. There is an urgent need to analyze the detailed polarization dependence from a theoretical model for radiation-induced magnetoresistance oscillations.

\section{MAGNETORESISTANCE UNDER POLARIZED RADIATION}

We deal with an isotropic $2 \mathrm{D}$ system of short thermalization time, consisting of $N_{s}$ electrons in a unit area of the $x-y$ plane. These electrons, scattered by random impurities and by phonons in the lattice, are subjected to a uniform magnetic field $\boldsymbol{B}=(0,0, B)$ in the $z$ direction. When an electromagnetic wave of angular frequency $\omega$ illuminates perpendicularly onto the $2 \mathrm{D}$ plane with the incident electric field

$$
\boldsymbol{E}_{\mathrm{i}}(t)=\boldsymbol{E}_{\mathrm{i} s} \sin (\omega t)+\boldsymbol{E}_{\mathrm{i} c} \cos (\omega t)
$$

at $z=0$ and a dc current flows within the plane, the electric field inside the 2D system involves a dc component, $\boldsymbol{E}_{0}$, and an ac component

$$
\boldsymbol{E}(t)=\boldsymbol{E}_{s} \sin (\omega t)+\boldsymbol{E}_{c} \cos (\omega t) .
$$

The steady-transport state of this electron system can be described by the drift velocity of the electron integrative (the center of mass) motion, consisting of a dc part, $\boldsymbol{v}$, and a stationary time-dependent part,

$$
\boldsymbol{v}(t)=\boldsymbol{v}_{s} \sin (\omega t)+\boldsymbol{v}_{c} \cos (\omega t),
$$

in the $2 \mathrm{D}$ plane, together with an average temperature $T_{e}$, characterizing the isotropic thermal distribution of electrons in the reference frame moving with the center 
of mass $\frac{36}{6}$ They satisfy the following force and energy balance equations: ${ }^{22}$

$$
\begin{gathered}
N_{s} e \boldsymbol{E}_{0}+N_{s} e(\boldsymbol{v} \times \boldsymbol{B})+\boldsymbol{F}=0, \\
\boldsymbol{v}_{c}=-\frac{e \boldsymbol{E}_{s}}{m \omega}-\frac{\boldsymbol{F}_{s}}{N_{s} m \omega}-\frac{e}{m \omega}\left(\boldsymbol{v}_{s} \times \boldsymbol{B}\right), \\
\boldsymbol{v}_{s}=\frac{e \boldsymbol{E}_{c}}{m \omega}+\frac{\boldsymbol{F}_{c}}{N_{s} m \omega}+\frac{e}{m \omega}\left(\boldsymbol{v}_{c} \times \boldsymbol{B}\right), \\
N_{s} e \boldsymbol{E}_{0} \cdot \boldsymbol{v}+S_{\mathrm{p}}-W=0 .
\end{gathered}
$$

Here,

$$
\boldsymbol{F}=\sum_{\boldsymbol{q}_{\|}}\left|U\left(q_{\|}\right)\right|^{2} \sum_{n=-\infty}^{\infty} \boldsymbol{q}_{\|} J_{n}^{2}(\xi) \Pi_{2}\left(q_{\|}, \omega_{0}-n \omega\right)
$$

is the time-averaged damping force against the electron drift motion due to impurity scattering, and

$$
S_{\mathrm{p}}=\sum_{\boldsymbol{q}_{\|}}\left|U\left(q_{\|}\right)\right|^{2} \sum_{n=-\infty}^{\infty} n \omega J_{n}^{2}(\xi) \Pi_{2}\left(q_{\|}, \omega_{0}-n \omega\right)
$$

is the time-averaged rate of the electron energyabsorption from the radiation field. In Eqs. (8) and (9), $U\left(q_{\|}\right)$is the effective impurity potential, $\Pi_{2}\left(q_{\|}, \Omega\right)$ is the imaginary part of the electron density-correlation function at temperature $T_{e}$ in the presence of the magnetic field without the electric field, $\omega_{0} \equiv \boldsymbol{q}_{\|} \cdot \boldsymbol{v}$, and $J_{n}(\xi)$ is the Bessel function of order $n$ with argument $\xi \equiv\left[\left(\boldsymbol{q}_{\|} \cdot \boldsymbol{v}_{s}\right)^{2}+\left(\boldsymbol{q}_{\|} \cdot \boldsymbol{v}_{c}\right)^{2}\right]^{\frac{1}{2}} / \omega$. Note that, although contributions of phonon scattering to $\boldsymbol{F}$ and $S_{\mathrm{p}}$ are neglected in comparison with those of impurity scattering at the considered low lattice temperature, it provides the main channel for electron energy dissipation to the lattice with a time-averaged energy-loss rate $W$, having an expression as given in Ref. 22 .

The ac components $\boldsymbol{v}_{s}$ and $\boldsymbol{v}_{c}$ of electron drift velocity should be determined selfconsistently in terms of the incident ac field $\boldsymbol{E}_{\mathrm{i}}(t)$ by the electrodynamic equations connecting both sides of the $2 \mathrm{D}$ system, taking into account the scattering-related damping forces $\boldsymbol{F}_{s}$ and $\boldsymbol{F}_{c}, 22$ However, for high-mobility systems at low temperatures, the effects of these scattering-related damping forces are much weaker in comparison to those of radiative decay $\underline{31}$ and thus negligible, whence $\boldsymbol{v}_{s}$ and $\boldsymbol{v}_{c}$ are in fact directly given from Eqs. (5) and (6) by the high-frequency electric field $\boldsymbol{E}(t)$ inside the 2D electron system. On the other hand, by solving the Maxwell equations connecting both sides of the $2 \mathrm{D}$ electron gas which is carrying the sheet current density $N_{s} e \boldsymbol{v}(t), \boldsymbol{E}(t)$ is determined by the incident fields $\boldsymbol{E}_{\text {is }}$ and $\boldsymbol{E}_{\mathrm{i} c}$ based on the setup of the 2D system in the sample substrate ${ }^{22}$

If the $2 \mathrm{D}$ electron gas locates within a thin layer under the surface plane at $z=0$ of a thick (treated as semi-infinite) semiconductor substrate having a refractive index, $n_{s}$, the ac field $\boldsymbol{E}(t)$ driving the 2D electrons, which equals the sum of the incident and the reflected fields at $z=0$ and equals the transmitted field (the field just passes through the 2D layer), can be expressed as ${ }^{37}$

$$
\boldsymbol{E}(t)=\frac{N_{s} e \boldsymbol{v}(t)}{\left(n_{0}+n_{s}\right) \epsilon_{0} c}+\frac{2 n_{0}}{n_{0}+n_{s}} \boldsymbol{E}_{\mathrm{i}}(t) .
$$

Here $n_{0}$ is the refractive index of the air and $c$ and $\epsilon_{0}$ are, respectively, the light speed and the dielectric constant in vacuum. If the $2 \mathrm{D}$ electron gas is contained in a thin layer suspended in vacuum at the plane $z=0$, then

$$
\boldsymbol{E}(t)=\frac{N_{s} e \boldsymbol{v}(t)}{2 \epsilon_{0} c}+\boldsymbol{E}_{\mathrm{i}}(t) .
$$

By combining Eq. (10) or Eq. (11) with balance Eqs. (5) and (6), the ac drift-velocity components $\boldsymbol{v}_{s}$ and $\boldsymbol{v}_{c}$, and thus the argument $\xi$, can be determined by the incident field components $\boldsymbol{E}_{\mathrm{i} s}$ and $\boldsymbol{E}_{\mathrm{ic}}$.

The imaginary part of $2 \mathrm{D}$ electron density correlation function in a magnetic field, $\Pi_{2}\left(q_{\|}, \Omega\right)$, can be written in the Landau representation as 38

$$
\begin{gathered}
\Pi_{2}\left(q_{\|}, \Omega\right)=\frac{1}{2 \pi l_{B}^{2}} \sum_{n, n^{\prime}} C_{n, n^{\prime}}\left(l_{B}^{2} q_{\|}^{2} / 2\right) \Pi_{2}\left(n, n^{\prime}, \Omega\right), \\
\Pi_{2}\left(n, n^{\prime}, \Omega\right)=-\frac{2}{\pi} \int d \varepsilon[f(\varepsilon)-f(\varepsilon+\Omega)] \\
\quad \times \operatorname{Im} G_{n}(\varepsilon+\Omega) \operatorname{Im} G_{n^{\prime}}(\varepsilon)
\end{gathered}
$$

where $l_{B}=\sqrt{1 /|e B|}$ is the magnetic length, $C_{n, n+l}(Y) \equiv$ $n ![(n+l) !]^{-1} Y^{l} \mathrm{e}^{-Y}\left[L_{n}^{l}(Y)\right]^{2}$ with $L_{n}^{l}(Y)$ being the associate Laguerre polynomial, $f(\varepsilon)=\left\{\exp \left[(\varepsilon-\mu) / T_{e}\right]+1\right\}^{-1}$ is the Fermi function at electron temperature $T_{e}$, and $\operatorname{Im} G_{n}(\varepsilon)$ is the density-of-states (DOS) function of the broadened Landau level $n$.

The Landau-level broadening, which results from impurity, phonon, and electron-electron scatterings, is assumed to have a Gaussian form $\left[\varepsilon_{n}=\left(n+\frac{1}{2}\right) \omega_{c}\right.$ is the center of the $n$th Landau level, $n=0,1,2, \ldots]$,

$$
\operatorname{Im} G_{n}(\varepsilon)=-(2 \pi)^{\frac{1}{2}} \Gamma^{-1} \exp \left[-2\left(\varepsilon-\varepsilon_{n}\right)^{2} / \Gamma^{2}\right],
$$

with a $B^{\frac{1}{2}}$-dependent half width expressed as

$$
\Gamma=\left(2 \omega_{c} / \pi \tau_{s}\right)^{\frac{1}{2}},
$$

where $\tau_{s}$, the single-particle lifetime or quantum scattering time in the zero magnetic field, is assumed to be related to the transport relaxation time $\tau_{\text {tr }}$ or the zerofield linear mobility $\mu_{0}$ using an empirical parameter $\alpha$ by ${ }^{22}$

$$
1 / \tau_{s}=4 \alpha / \tau_{\text {tr }}=4 \alpha e / m \mu_{0} .
$$

For an isotropic system where the frictional force $\boldsymbol{F}$ is in the opposite direction of the drift velocity $\boldsymbol{v}$ and the magnitudes of both the frictional force and the energydissipation rate depend only on $v \equiv|\boldsymbol{v}|$, we can write $\boldsymbol{F}(\boldsymbol{v})=F(v) \boldsymbol{v} / v$ and $W(\boldsymbol{v})=W(v)$. In the Hall configuration with velocity $\boldsymbol{v}$ in the $x$ direction $\boldsymbol{v}=(v, 0,0)$ 


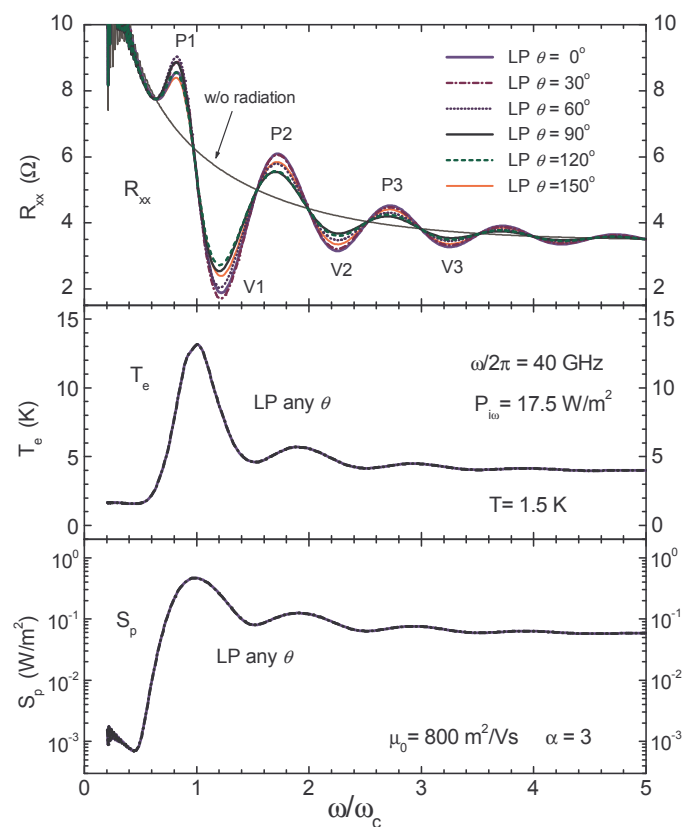

FIG. 1: (Color online) Magnetoresistivity $R_{x x}$, electron temperature $T_{e}$ and energy absorption $S_{\mathrm{p}}$ are plotted versus $\omega / \omega_{c}$ under the irradiation of linearly polarized (LP) microwaves of frequency $40-\mathrm{GHz}$ and incident power $P_{\mathrm{i} \omega}=$ $17.5 \mathrm{~W} / \mathrm{m}^{2}$ at several different polarization directions $(\theta=$ $0^{\circ}, 30^{\circ}, 60^{\circ}, 90^{\circ}, 120^{\circ}$ and $150^{\circ}$ ) for a system described in the text at temperature $T=1.5 \mathrm{~K}$.

or the current density $J_{x}=J=N_{s} e v$, and $J_{y}=0$, the longitudinal linear $(v \rightarrow 0)$ resistivity under the incident radiation of Eq. (1) due to impurity scattering, can be written in the form

$$
R_{x x}=-\sum_{\boldsymbol{q}_{\|}} q_{x}^{2} \frac{\left|U\left(q_{\|}\right)\right|^{2}}{N_{s}^{2} e^{2}} \sum_{n=-\infty}^{\infty} J_{n}^{2}(\xi) \Pi_{2}^{\prime}\left(q_{\|}, n \omega\right),
$$

where $\Pi_{2}^{\prime}\left(q_{\|}, \Omega\right) \equiv \partial \Pi_{2}\left(q_{\|}, \Omega\right) / \partial \Omega$.

\section{OSCILLATORY RESISTIVITY VERSUS POLARIZATION DIRECTION}

We consider an incident radiation of form (1) linearly polarized along the direction having an angle $\theta$ with respect to the $x$-axis: $\boldsymbol{E}_{\mathrm{i} s}=\left(E_{\mathrm{i} \omega} \cos \theta, E_{\mathrm{i} \omega} \sin \theta\right)$ and $\boldsymbol{E}_{\mathrm{ic}}=0$. The 2D electron gas, having carrier sheet density $N_{s}=2.2 \times 10^{15} \mathrm{~m}^{-2}$ and zero-temperature linear mobility $\mu_{0}=800 \mathrm{~m}^{2} / \mathrm{V}$ s from short-range impurity scattering in the absence of the magnetic field, is assumed to locate within a thin layer under the surface plane at $z=0$ of a thick GaAs-based substrate with a refractive index of $n_{s}=3.59$.

Figure 1 shows the calculated energy absorption rate $S_{\mathrm{p}}$, the electron temperature $T_{e}$ and the longitudinal linear resistivity $R_{x x}$ of this system irradiated by linearly polarized (LP) incident microwaves having frequency
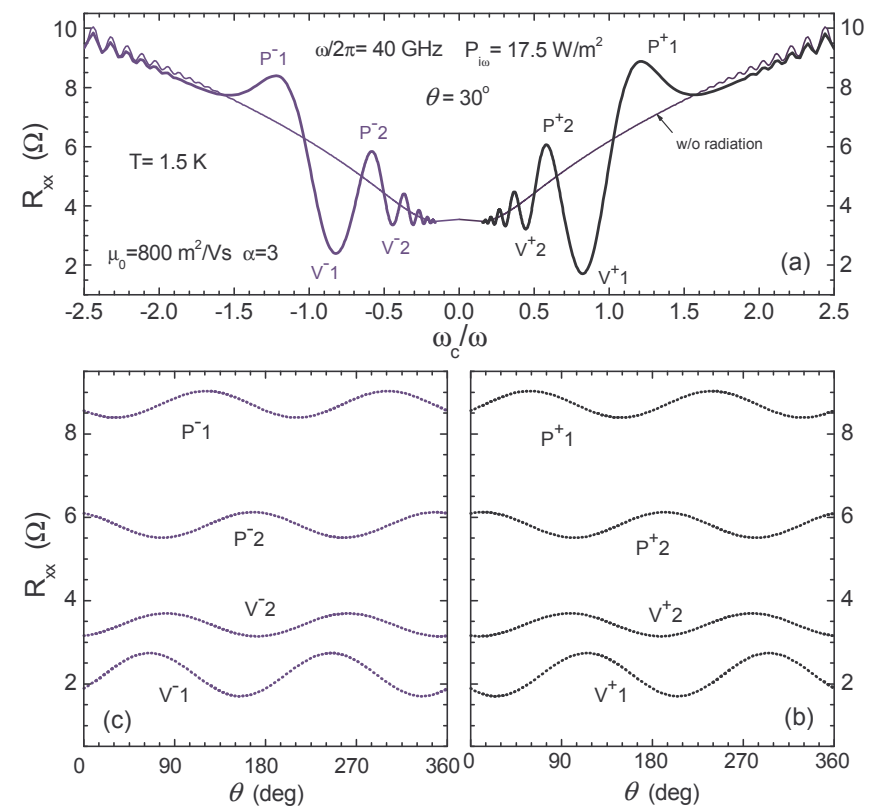

FIG. 2: (Color online) Magnetoresistivity $R_{x x}$ induced by linearly polarized microwave of frequency $40 \mathrm{GHz}$ and incident power $P_{\mathrm{i} \omega}=17.5 \mathrm{~W} / \mathrm{m}^{2}$ at $\theta=30^{\circ}$ is shown for positive and reverse magnetic fields over the range $-2.5 \leq \omega_{c} / \omega \leq 2.5$ (a). The $\theta$ dependence of $R_{x x}$ at extrema $P^{+} 1, V^{+} 1, P^{+} 2$, and $V^{+} 2$ (b) and at extrema $P^{-} 1, V^{-} 1, P^{-} 2$, and $V^{-} 2$ (c).

$\omega / 2 \pi=40 \mathrm{GHz}$ and amplitude $E_{\mathrm{i} \omega}=1.15 \mathrm{~V} / \mathrm{cm}$ (i.e., incident power $\left.P_{\mathrm{i} \omega}=17.5 \mathrm{~W} / \mathrm{m}^{2}\right)$ at different polarization directions $\left(\theta=0^{\circ}, 30^{\circ}, 60^{\circ}, 90^{\circ}, 120^{\circ}\right.$, and $\left.150^{\circ}\right)$, together with the dark resistivity, as functions of the normalized inverse magnetic field $\omega / \omega_{c}\left(\omega_{c}=e B / m\right)$. The lattice temperature is assumed to be $T=1.5 \mathrm{~K}$ and the Landau level broadening parameter is taken to be $\alpha=3$.

We see that the electron energy absorption $S_{\mathrm{p}}$ and thus the electron temperature $T_{e}$, both showing a marked main peak at cyclotron resonance $\omega / \omega_{c}=1$ and secondary peaks around its harmonics $\omega / \omega_{c}=2,3,4, \ldots$, are essentially the same for all different polarization directions of radiation. This can be understood directly from expression (9) of $S_{\mathrm{p}}$ in the case of relatively low strength of incident microwave when the dominant contribution to it comes from the terms $n= \pm 1$ and $J_{ \pm 1}^{2}(\xi) \sim \xi^{2}$. Writing explicitly the $\theta$-dependent expression of $\xi^{2}$ in the case of incident plane-polarized radiation having an angle $\theta$ with respect to the $x$-axis we can see that, after summing over all the directions of $\boldsymbol{q}_{\|}$, the angle dependence disappears due to $\sin ^{2} \theta+\cos ^{2} \theta=1$. Furthermore, the energy-loss rate $W$ has $\theta$-dependent behavior similar to that $S_{\mathrm{p}}$. Thus the electron temperature $T_{e}$, determined by the energy-balance equation (7) involving only $S_{\mathrm{p}}$ and $W$, has similar $\theta$-dependent behavior. The situation is different for the frictional force $\boldsymbol{F}$ and the resistivity $R_{x x}$, because of additional $\boldsymbol{q}_{\|}$-direction dependent weighted factors showing up inside the $\boldsymbol{q}_{\|}$summation in the expressions (8) and (17), leading to sensitive $\theta$-dependence 
of $R_{x x}$ as seen from the numerical results shown in the upper part of Fig. 1. The linear-polarized microwave excited magnetoresistivity $R_{x x}$ oscillates strongly, having the same oscillatory period and nearly the same phase and, at cyclotron resonance $\omega / \omega_{c}=1$ or its harmonics $\omega / \omega_{c}=2,3,4, \ldots$, taking equal values for all different polarization directions $\theta$. We can label, for all $\theta$, the main peaks and valleys of the oscillatory resistivity respectively as $P 1, P 2$, and $V 1, V 2$, etc. The amplitude of resistivity oscillation, however, varies sensitively with changing the polarization direction of the microwave. The maximal or minimal amplitude of a peak or a valley shows up at different polarization angles $\theta$ for different peaks or valleys. For instance, the maximal and minimal amplitudes show up, respectively, at $\theta \approx 59^{\circ}$ and $\theta \approx 149^{\circ}$ for $P 1$, at $\theta \approx 25^{\circ}$ and $\theta \approx 115^{\circ}$ for $V 1$, at $\theta \approx 12^{\circ}$ and $\theta \approx 102^{\circ}$ for $P 2$, at $\theta \approx 8^{\circ}$ and $\theta \approx 98^{\circ}$ for $V 2$, and show up at polarization angles closer to $\theta=0^{\circ}$ and $\theta=90^{\circ}$ for higher order peaks and valleys. The exact polarization angle for the maximal or minimal amplitude of an extremum appears to depend on the radiation frequency and the $2 \mathrm{D}$ electron setup in the substrate. In obtaining the above results the sample setup is so assumed that $2 \mathrm{D}$ electrons locate within a thin layer under the surface plane at $z=0$ of a thick semiconductor substrate having a refractive index of $n_{s}=3.59$, and the relevant damping in question, i.e., the radiative decay, is fully determined by this sample setup from the electrodynamic equation (10). Except for this, no other damping parameter nor any mechanism capable of producing polarization rotation is introduced in the present model.

Next we present the results obtained under magnetic field reversal. Figure 2(a) plots the longitudinal resistivity $R_{x x}$ vs $\omega_{c} / \omega$ over the range $-2.5 \leq \omega_{c} / \omega \leq 2.5$ for the system irradiated by a linearly polarized microwave along the $\theta=30^{\circ}$ direction with frequency $\omega / 2 \pi=40 \mathrm{GHz}$ and incident power $P_{\mathrm{i} \omega}=17.5 \mathrm{~W} / \mathrm{m}^{2}$. The extrema of interest here are labeled as $P^{+} 1, V^{+} 1, P^{+} 2$, and $V^{+} 2$ for those in the domain $B>0$, and $P^{-} 1, V^{-} 1, P^{-} 2$, and $V^{-} 2$ for those in the domain $B<0$. The values of longitudinal resistivity $R_{x x}$ at $P^{+} 1, V^{+} 1, P^{+} 2$, and $V^{+} 2$ are plotted in Fig. 2(b), and those at $P^{-} 1, V^{-} 1, P^{-} 2$, and $V^{-} 2$ qre plotted in Fig. 2(c), as functions of the microwave polarization direction $\theta$. The present treatment is for an isotropic system. By symmetry we always have $P^{+} 1(\theta)=P^{+} 1(\pi+\theta), P^{-} 1(\theta)=P^{-} 1(\pi+\theta)$, $P^{+} 1(\theta)=P^{-} 1(\pi-\theta)$, etc. If the maximal amplitude of an extremum, e.g., $P^{+} 1$, shows up at $\theta \neq 0$, the maximal amplitude of the corresponding extremum at the reverse magnetic field, $P^{-} 1$, must be at a different polarization angle. The effect of asymmetry in a real sample itself would produce further complexity.

\section{SUMMARY}

We have examined the effect of changing the polarization angle $\theta$ of the incident linearly-polarized microwaves with respect to the dc current on radiation-induced magnetoresistance oscillations within the balance-equation formulation of the photon-assisted magnetotransport model.

The present investigation takes the incident microwave field, rather than the ac field inside the $2 \mathrm{D}$ electron system, as the input quantity, allowing a direct determination of the dominant damping mechanism in the highmobility system, the radiative decay, from the experimental sample setup without introducing any artificial damping parameter, thus enabling a direct comparison with experimental measurement.

We find that the amplitude of radiation-induced magnetoresistance oscillation varies sensitively with changing $\theta$. At an extremum the amplitude of oscillatory magnetoresistance $R_{x x}$ exhibits a sinusoidal, up to a factor of 5 , magnitude variation with rotating the polarization angle $\theta$. The maximal amplitude shows up generally at a nonzero $\theta$, which is dependent upon the extremum in question, the $2 \mathrm{D}$ electron setup, the radiation frequency and the magnetic field orientation.

This work was supported by the National Science Foundation of China (Grant No. 61131006) and the National Basic Research Program of China (Grant No. 2011CB925603).
1 M. A. Zudov, R. R. Du, J. A. Simmons, J. L. Reno, Phys. Rev. B 64, 201311(R) (2001).

${ }^{2}$ P. D. Ye, L. W. Engel, D. C. Tsui, J. A. Simmons, J. R. Wendt, G. A. Vawter, and J. L. Reno, Appl. Phys. Lett. 79, 2193 (2001).

3 R. G. Mani, J. H. Smet, K. von Klitzing, V. Narayanamurti, W. B. Johnson, and V. Umansky, Nature (London) 420, 646 (2002); Phys. Rev. Lett. 92, 146801 (2004).

${ }^{4}$ M. A. Zudov, R. R. Du, L. N. Pfeiffer, and K. W. West, Phys. Rev. Lett. 90, 046807 (2003); ibid. 96, 236804 (2006); Phys. Rev. B 73, 041303(R) (2006).

5 S. I. Dorozhkin, JETP Lett. 77, 577 (2003).

${ }^{6}$ C. L. Yang, M. A. Zudov, T. A. Knuuttila, R. R. Du, L. N. Pfeiffer, and K. W. West, Phys. Rev. Lett. 91, 096803
(2003).

7 M. A. Zudov, Phys. Rev. B 69, 041304(R) (2004).

8 R. G. Mani, V. Narayanamurti, K. von Klitzing, J. H. Smet, W. B. Johnson, and V. Umansky, Phys. Rev. B 70, 155310 (2004).

9 R. G. Mani, Phys. E 22, 1 (2004); Phys. Rev. B 72, 075327 (2005); Appl. Phys. Lett. 85, 4962 (2004); ibid. 91, 132103 (2007).

10 R. L. Willett, L. N. Pfeiffer, and K. W. West, Phys. Rev. Lett, 93, 026804 (2004)

11 S. I. Dorozhkin, J. H. Smet, V. Umansky, K. von Klitzing, Phys. Rev. B 71, 201306(R) (2005).

12 S. A. Studenikin, M. Potemski, A. Sachrajda, M. Hilke, L. N. Pfeiffer, and K. W. West, IEEE Trans. Nanotechnol. 
4, 124 (2005); Phys. Rev. B 71, 245313 (2005).

13 J. H. Smet, B. Gorshunov, C. Jiang, L. N. Pfeiffer, K. W. West, V. Umansky, M. Dressel, R. Meisels, F. Kuchar, and K. von Klitzing, Phys. Rev. Lett. 95, 116804 (2005).

14 C. L. Yang, R. R. Du, L. N. Pfeiffer, and K. W. West, Phys. Rev. B 74, 045315 (2006).

15 A. T. Hatke, M. A. Zudov, L. N. Pfeiffer, and K. W. West, Phys. Rev. Lett. 102, 066804 (2009); Phys. Rev. B 79, 161308(R) (2009).

16 R. G. Mani, C. Gerl, S. Schmult, W. Wegscheider, and V. Umansky, Phys. Rev. B 81, 125320 (2010).

17 R. G. Mani, A. N. Ramanayaka, and W. Wegscheider, Phys. Rev. B 84, 085308 (2011).

18 A. N. Ramanayaka, R. G. Mani, J. Iñarrea, and W. Wegscheider, Phys. Rev. B 85, 205315 (2012).

19 V. I. Ryzhii and R. Suris, J. Phys. Condens. Matter 15, 6855 (2003); V. I. Ryzhii and V. Vyurkov, Phys. Rev. B 68, 165406 (2003); V. I. Ryzhii, Jpn. J. Appl. Phys., Part 1 44, 6600 (2005).

20 J. Shi and X. C. Xie, Phys. Rev. Lett. 91, 086801 (2003).

21 A. C. Durst, S. Sachdev, N. Read, and S. M. Girvin, Phys. Rev. Lett. 91, 086803 (2003).

22 X. L. Lei and S. Y. Liu, Phys. Rev. Lett. 91, 226805 (2003); Phys. Rev. B 72, 075345 (2005); Appl. Phys. Lett. 86, 262101 (2005); X. L. Lei, J. Phys. Condens. Matter 16, 4045 (2004).

23 A. A. Koulakov and M. E. Raikh, Phys. Rev. B 68, 115324 (2003).

24 M. G. Vavilov and I. L. Aleiner, Phys. Rev. B 69, 035303 (2004).

25 M. Torres and A. Kunold, Phys. Rev. B 71, 115313 (2005);
J. Phys. Condens. Matter 18, 4029 (2006).

26 T. K. Ng and Lixin Dai, Phys. Rev. B 72, 235333 (2005).

27 J. Iñarrea and G. Platero, Phys. Rev. Lett. 94, 016806 (2005); Phys. Rev. B 72, 193414 (2005).

28 A. Kashuba, Phys. Rev. B 73, 125340 (2006).

29 I. A. Dmitriev, A. D. Mirlin, and D. G. Polyakov, Phys. Rev. Lett. 91, 226802 (2003); 99, 206805 (2007); Phys. Rev. B 70, 165305 (2004); 75, 245320 (2007); I. A. Dmitriev, M. G. Vavilov, I. L. Aleiner, A. D. Mirlin, and D. G. Polyakov, ibid. 71, 115316 (2005).

30 J. Dietel, L. I. Glazman, F. W. J. Hekking, and F. von Oppen, Phys. Rev. B 71, 045329 (2005); C. Joas, J. Dietel, and F. von Oppen, ibid. 72, 165323 (2005); J. Dietel, ibid. 73, 125350 (2006).

31 S. A. Mikhailov, Phys. Rev. B 70, 165311 (2004).

32 X. L. Lei and S. Y. Liu, Appl. Phys. Lett. 88, 212109 (2006); ibid. 89, 182117 (2006); ibid. 94, 232107 (2009).

33 J. Iñarrea and G. Platero, Phys. Rev. B 76, 073311 (2007); J. Iñarrea, Appl. Phys. Lett. 100, 242103 (2012).

34 S. S. Wang and T. K. Ng, Phys. Rev. B 77, 165324 (2008).

35 I. A. Dmitriev, M. Khodas, A. D. Mirlin, D. G. Polyakov, and M. G. Vavilov, Phys. Rev. B 80, 165327 (2009).

36 X. L. Lei and C. S. Ting, Phys. Rev. B 30, 4809 (1984); ibid, 32, 1112 (1985); X. L. Lei, J. L. Birman, and C. S. Ting, J. Appl. Phys. 58, 2270 (1985).

37 K. W. Chiu, T. K. Lee, and J. J. Quinn, Surf. Sci. 58, 182 (1976).

38 C. S. Ting, S. C. Ying, and J. J. Quinn, Phys. Rev. B 16, 5394 (1977). 